\title{
Embedded Energy and Power Management System
}

\author{
K.Bharathi \\ PG Student \\ Sri Venkatesa Perumal College \\ of Engineering
}

\author{
M.Jasmine \\ Associate Professor \\ Sri Venkatesa Perumal College \\ of Engineering
}

\author{
Madhu Nakirekanti \\ Assistant Professor \\ Vardhaman College of \\ Engineering
}

\begin{abstract}
In this paper, Embedded Energy and Power Management System (EEPMS) to manage power consumption, automatic meter reading in home area is described. The EEPMS is a single phase digital $\mathrm{kWh}$ power meter with embedded Zigbee modem which utilize the Wireless sensor network to send its power usage reading using information back to the energy provider wirelessly. At the power provider side they have the control to change priority of the devices when power distributed in low range. The concept of dynamic assignment of priorities to interrupts is discussed which reduces the time delay for a lower priority task which under some circumstances becomes a higher priority task. Slit of interrupt timings is also observed which can be used to improve the performance.
\end{abstract}

\section{Keywords}

EEPMS, Zigbee, Power meter, priority, WSN.

\section{INTRODUCTION}

The implementation of these energy saving technologies needs meticulous measurement of consumptions, because effective measurement is equal to pompous performance of the coherent technologies. For this reason only the demand increasing form this energy and power measuring instruments. The growth of technology leads the usage power increased more in the home area. Although advanced integrated circuit (IC) chipset and hardware technology magnifies the power efficiency of home appliances and consumer electronics, the current energy crisis and green house effect require energy and power management in all areas. In this described system, we have designed an architecture of effective power management in the home section, which can be controlled by a home server[1]. The home server is linked with the home section by means of Wireless sensor network (WSN)[2-3].

In this paper, we bring forward more efficient EEPMS based on ZigBee communication and ARM processor. To implement the automatic standby power cut-off outlet with power measurement functions[4-5]. The home server is designed to collect the information about the control section and at the same time the consumed power will be displayed in the control section itself.

In this architecture, we proposed a clear management system, which is having a priority based control system. This is nothing but, when the server sends the command signal then the unit will go to the particular priority mode. So that wastage of power will be prevented.

\section{EEPMS ARCHITECTURE}

The architecture of the proposed EEPMS Architecture is as shown in Fig 1(a\&b). The proposed architecture uses a processor as a heart of the system and the power management priority settings will be programmed. Current transformer is provided to sense the exact current flowing in distributed power line. Voltage transformer is provided to measure the voltage in that line. Signal conditioning unit is used give an equivalent voltage and current to the micro controller unit The information will be processed by the processor and it will calculate the amount for that consumed power. For consumer purpose this information will be display on the LCD. So Automatic power meter reading done with this EEPMS System and this system having the load sharing part based on priority to control the power consumption.

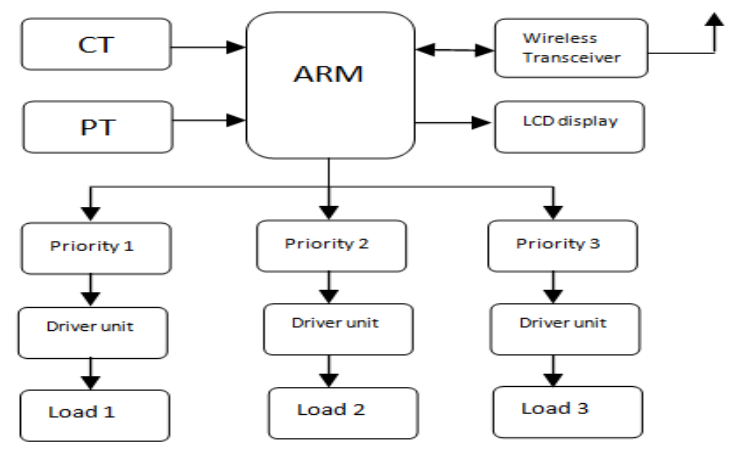

(a)

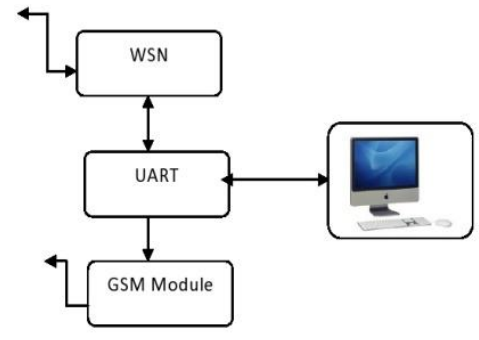

(b)

Figure 1 (a) EEPMS architecture, (b). Home server Section

The server room section is shown on Figure.1 (b).Here, the GSM and Zigbee connected to the Server using UART. ZigBee modem in EEPMS continuously transmits the data and the Zigbee modem in the Server Room receives the data

The amount level also displays on the server control screen and also sends amount levels, Due date as SMS to the consumer by using GSM modem. So that the user can know the amount level and due date. After completion of the due date also if the consumer didn't pay the amount the server room can send a control signal to the EEPMS to open the relay. Then automatic power cut can achieve with this system.

In implementation we are sending the signal from the home server using ZigBee as a Transmitter, In the Receiver part another ZigBee connected to the EEPMS. After receiving the signal ARM checks the priority and sends an interrupt to the connected relays. According to the priority interrupt the Load will change. 


\section{IMPLEMENTATION AND RESULTS}

In this system we are using ARM processor as a heart and WSN communication as a transmission and receiving medium for the data transfer and receive. In this EEPMS there are two important sections. One is EEPMS hardware section and the next one is server section. The two sections are connected with the help of Zigbee transceivers. The processor is connected to the CT and PT, which gives the individual current and voltage value to the processor. The processor calculate power factor and amount according to the given program to the processor and displays on the connected LCD to EEPMS section. We are selecting the option in the server control screen as shown in following figure and sending the signal from the server room using ZigBee as a Transmitter, In the Receiver part another ZigBee connected to the EEPMS. After receiving the signal from the Home server it sends to the signal to the ARM7 processor, after receiving the signal ARM7 sends an interrupt to the connected relays. According to the priority interrupt the Load will change[6-9].

The figure shows the EEPMS monitor and control section. The controller can monitor the current power consumption at the same time according to the requirement we can control power distribution.

The home server display window has the feasibility to show the amount and energy consumption. The Server can show the customer ID, customer name, energy consumed and amount of charge for consumed energy and due date.According to the program the amount and Energy will be calculated and displayed on the LCD.By connecting Zigbee through UART communication this data will transfer to PC.

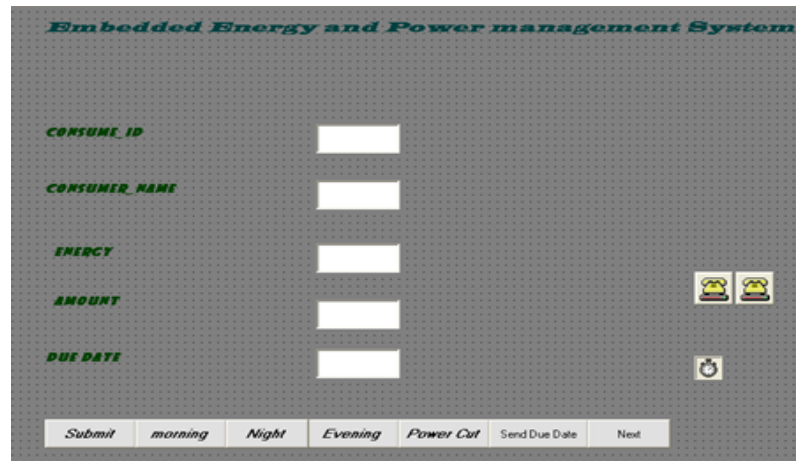

Figure 2: Captured display of Home server control screen

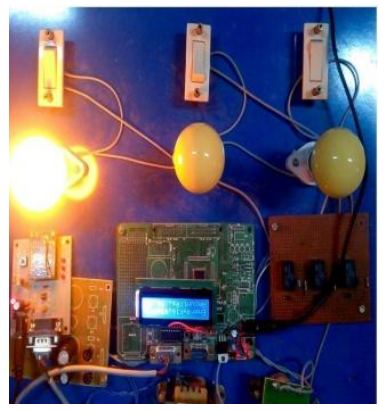

(a)

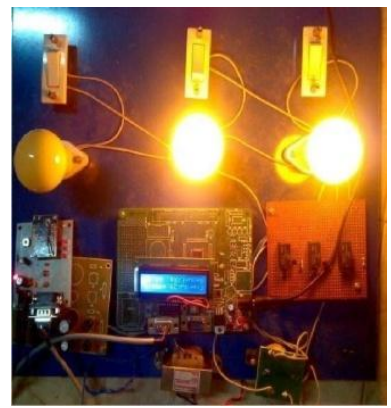

(b)

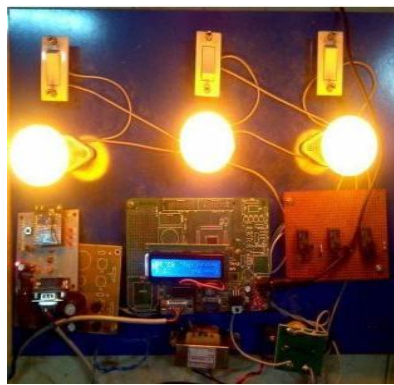

(c)

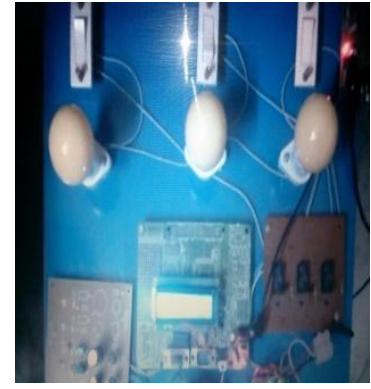

(d)
Figure 3: Implemented boards for (a) Morning (b) After noon (c) Evening/Power full (d) Power cut

From this total operation can control the power consumption and can achieve the uninterrupted power supply to the consumer by checking the power storage available for the home server. If storage is insufficient, have to select Morning, in this priority the load is connected to essential needs of consumer like one bulb, one fan etc.. If the storage is moderate have to select Afternoon, in this the load is connected to bulbs, fans, cooker etc.. If enough storage is available for the home serve selecting power full /Evening, in this the load is connected to all the electric appliances. If the operator want to cut the power to the particular customer if he is not paid the bill he have to select power cut priority as shown in the Figure

\section{CONCLUSION}

Nowadays Power management is going to be essential day to day life. In order to make the power saving EEPMS is playing an effective role to bring the power wastage factor of control. In order to make the power saving IEPMS is playing an effective role to bring the power wastage factor in control. According to the requirements the control commands will be passed to the IEPMS device which will turn on or off the load with respect to the priority. Thus the power can be managed in an efficient way.

\section{REFERENCES}

[1] Giovanni Bucci, Edoardo Fiorucci,Fabrizio Ciancetta, Daniele Gallo, Carmine Landi, Mario Luiso "Embedded Power and Energy Measurement System Based on an Analog Multiplier" IEEE Trans on Instrumentation and measurement, Vol 62,No 8,pp. 2248-2257, Aug 2013.

[2] Jinsoo Han, Chang-Sic Choi, Ilwoo Lee "More efficient home energy management system based on ZigBee communication and infrared remote controls" IEEE Trans on Consumer Electronics, Vol 57,No 1,pp. 8589,Feb 2011.

[3] Insung Hong, Byeongkwan Kang, and Sehyun Park, "Design and Implementation of Intelligent Energy Distribution Management with Photovoltaic System" IEEE Trans on Consumer Electronics, Vol. 58, No. 2,pp.340-346 May 2012.

[4] Hou Weiyan, Wang Jiahui, Zhang Fangchang "A Scheme for the Application of Smart Message Language in a Wireless Meter Reading System" Measuring Technology and Mechatronics Automation (ICMTMA), $3^{\text {rd }}$ International Conference on Vol 1.pp.254-257,jan 2011. 
[5] PrudhviPotuganti. Bhalodi,Manohar.M, Padidela.V,Suda rshanAdapa"A Smart energy Meter Architecture in India n context" 11th International Conference on Environment and Electrical Engineering (EEEIC), pp.217-222, may2012.

[6] Adeel A. Siddiqui, Arbab Waheed Ahmad, Hee Kwon Yang, Chankil Lee "ZigBee Based Energy Efficient Outdoor Lighting Control System" $14^{\text {th }}$ International Conference on Advanced Comm. Technology (ICACT),pp.916-919,Feb2012.
[7] A. Collins, Solid State Solutions for Electricity Metrology, Metering and Tariffs for Energy Supply, no. 462, May 1999.

[8] Shang-Wen Luan,Jen-Hao Teng, Shun-Yu Chan; LainChyr Hwang,"Development of a smart power meter for AMI based on ZigBee communication" Power Electronics and Drive Systems, 2009. PEDS 2009. International Conference on , vol. no., pp.661-665, Nov 2009.

[9] GE Company, "Energy Efficiency Comparisons of Wireless Communication Technology Options for Smart Grid Enabled Devices,” Dec. 2010. 